\title{
Efeito da fluidoterapia transcirúrgica sobre o hematócrito pós-cirúrgico em cadelas submetidas a ovariossalpingo-histerectomia eletiva
}

\author{
[Effects of trans surgical fluid therapy over the post surgical packed cell volume \\ in bitches undergoing elective ovariohisterectomy] \\ J.T. Druziani ${ }^{1}$, F.B. Fukushima ${ }^{2}$, A.V. Gomes ${ }^{3}$, G.B. Quinaglia ${ }^{1}$, J.F. Vizzu ${ }^{1}$, \\ O. Cunha ${ }^{2}$, J.O. Sacchi ${ }^{1}$, M.M. Silva ${ }^{2}$ \\ ${ }^{1}$ Hospital Veterinário da Universidade Federal do Paraná - Setor Palotina, PR \\ ${ }^{2}$ Universidade Federal do Paraná - Setor Palotina, PR \\ ${ }^{3}$ Aluno de graduação - Universidade Federal do Paraná - PR
}

\begin{abstract}
RESUMO
Apesar dos inúmeros benefícios da fluidoterapia transcirúrgica, sobrecarga de volume pode trazer efeitos deletérios, como a alteração de parâmetros hematimétricos. Dessa maneira, o presente trabalho teve como objetivo avaliar duas diferentes taxas de infusão de solução cristaloide no período transanestésico e seus efeitos até 24 horas pós-operatório. Foram utilizados dois grupos de seis animais cada, um deles recebeu solução de cloreto de sódio $0,9 \%$ a $10 \mathrm{~mL} / \mathrm{kg} / \mathrm{h}(\mathrm{G} 10)$ e o outro a $5 \mathrm{~mL} / \mathrm{kg} / \mathrm{h}(\mathrm{G} 5)$. Os valores de hematócrito foram avaliados no período de 24 horas em 10 diferentes momentos. Os resultados não apontaram diferenças significativas entre os grupos, porém foi observada redução significativa do hematócrito após indução anestésica. Foi ainda observada redução de hematócrito após o término da cirurgia em ambos os grupos, e o retorno aos valores basais de hematócrito ocorreu de forma significativa 12 horas após o procedimento cirúrgico em G10, e após oito horas em G5, mostrando uma tendência à hemodiluição mais persistente em G10.
\end{abstract}

Palavras-chave: fluido, anestesia, castração, hematologia, hemodiluição

\begin{abstract}
Despite the beneficial goals of fluid therapy administered during surgery, volume overload can cause deleterious effects, such as alterations on hematimetric parameters. Thus, the objective of this paper was to evaluate two different cristaloid infusion rates during the surgical period and its effects on the 24-hour post-surgical period. Two groups of six animals each were used in the present study, one received $10 \mathrm{~mL} / \mathrm{kg} / \mathrm{h}(\mathrm{G} 10)$ of $0,9 \%$ sodium chloride solution and the other $5 \mathrm{~mL} / \mathrm{kg} / \mathrm{h}$ (G5) of the same solution. Packed cell volume (PCV) was evaluated in 10 different times during the 24 hours following surgery. The results did not show significant differences between groups, but they showed a major tendency of hemodilution in G10. A significant decrease of PCV was observed after induction of anesthesia. Decrease of PCV after the end of surgery in both groups was also observed, and the return to PCV basal values was observed 12 hours after the procedure in G10 and after eight hours in G5, showing a tendency of prolonged hemodilution in G10.
\end{abstract}

Keywords: fluid, anesthesia, neutering, hemathology, hemodilution

\section{INTRODUÇÃO}

$\mathrm{O}$ estabelecimento e a manutenção de acesso venoso são de extrema importância no período transanestésico. A fluidoterapia tem como

Recebido em 3 de agosto de 2016

Aceito em 10 de outubro de 2016

E-mail: julianatdruzi@gmail.com funções corrigir perdas ocasionadas pelo jejum pré-anestésico e pelo procedimento cirúrgico propriamente dito, manter um acesso venoso patente e tratar os danos causados pelos agentes anestésicos, como hipotensão. Assim, a taxa de fluidoterapia deve variar de acordo com a 
condição clínica do paciente, a duração e o tipo de procedimento a ser realizado (Davis et al., 2013).

A taxa de fluidoterapia com cristaloides anteriormente recomendada para cães hígidos submetidos à anestesia geral variava de 5 a $10 \mathrm{~mL} / \mathrm{kg} / \mathrm{h}$ (Hall et al., 2001), mas a tendência atual é a redução para valores menores quando não se espera perda de grande quantidade de fluido no perioperatório (Chohan e Davidow, 2015). De acordo com as novas diretrizes da American Animal Hospital Association (AAHA), taxas de 3 e $5 \mathrm{~mL} / \mathrm{kg} / \mathrm{h}$ para gatos e cães, respectivamente, são suficientes para reposição de pequenas perdas em pacientes submetidos a procedimentos eletivos (Davis et al., 2013).

A sobrecarga de volume de fluido durante o procedimento anestésico está associada a problemas como hipoalbuminemia e queda do valor de hematócrito (Mathews, 2006; AguilarNascimento et al., 2012), edema pulmonar e intersticial, aumento de peso corpóreo, coagulopatias, maiores taxas de infecção, diminuição de temperatura corporal, diminuição de motilidade intestinal, falência renal, maior tempo até alta hospitalar e hemodiluição (Chappell et al., 2008). O uso de altas taxas de fluidoterapia transcirúrgica pode ainda resultar em lesões de hipóxia por mais de 72 horas (Funk e Baldinger, 1995).

Apesar de a literatura ser vasta na avaliação do efeito da fluidoterapia em condições patológicas, não há trabalhos até o momento que avaliem o efeito prolongado de diferentes taxas de fluidoterapia em pacientes saudáveis submetidos a procedimento de curta duração.

Assim, considerando a importância da fluidoterapia transanestésica com cristaloides, este trabalho tem como objetivo avaliar o efeito hemodiluidor da fluidoterapia transcirúrgica a $5 \mathrm{~mL} / \mathrm{kg} / \mathrm{h}$ e $10 \mathrm{~mL} / \mathrm{kg} / \mathrm{h}$ durante 24 horas pósovariossalpingo-histerectomia eletiva em cadelas.

\section{MATERIAL E MÉTODOS}

Foram utilizadas 12 cadelas adultas, com idade entre um e seis anos, pesando entre oito e $36 \mathrm{~kg}$ $(14,5 \pm 7,9)$. Todos os animais foram provenientes do projeto de extensão "Controle populacional de cães e gatos e conscientização da posse responsável no contexto social das populações carentes". Para inclusão no estudo, todas as cadelas foram previamente submetidas a exame físico e hemograma, e aquelas consideradas saudáveis foram submetidas a ovariossalpingohisterectomia eletiva, após autorização do tutor por meio de assinatura do Termo de Consentimento Livre e Esclarecido (TCLE).

Os animais foram aleatoriamente distribuídos em dois grupos experimentais com seis animais cada. Ambos os grupos receberam solução de cloreto de sódio a $0,9 \%$ (Ecoflac® plus, solução de cloreto de sódio 0,9\%, B Braun, Brasil) durante o período transcirúrgico. $\mathrm{O}$ grupo $\mathrm{G} 10$ recebeu infusão de $10 \mathrm{~mL} / \mathrm{kg} / \mathrm{h}$ e o grupo G5, $5 \mathrm{~mL} / \mathrm{kg} / \mathrm{h}$. Todos os animais foram submetidos a jejum alimentar e hídrico de oito horas. A medicação pré-anestésica (MPA) foi realizada com maleato de acepromazina $0,2 \%$ (Apromazin ${ }^{\circ}$, maleato de acepromazina 0,2\%, Syntec, Brasil) $(0,04 \mathrm{mg} / \mathrm{kg})$, associada a sulfato de morfina (sulfato de morfina $10 \mathrm{mg} / \mathrm{mL}$, Hipolabor, Brasil) $(0,7 \mathrm{mg} / \mathrm{kg})$, ambos por via intramuscular. A indução anestésica foi realizada com propofol (Propovan ${ }^{\circledR}$, propofol $1 \%$, Cristália, Brasil) dose-efeito e midazolam (Dormire®, midazolam, Cristália, Brasil) $(0,2 \mathrm{mg} / \mathrm{kg})$, por via intravenosa. Os pacientes foram submetidos a bloqueio peridural com cloridrato de lidocaína $2 \%$ sem vasoconstritor (Lidocaína 2\%, Hipolabor, Brasil) e sulfato de morfina $(0,1 \mathrm{mg} / \mathrm{kg})$, com volume total de $0,25 \mathrm{~mL} / \mathrm{kg}$.

A manutenção anestésica foi realizada com isoflurano (Isoflurano, BioChimico, Brasil) suficiente para manutenção do plano anestésico cirúrgico e $100 \%$ de oxigênio. No período transanestésico, avaliaram-se frequência cardíaca e saturação de oxi-hemoglobina por oxímetro de pulso, frequência respiratória mediante observação de movimentos torácicos, pressão arterial não invasiva por oscilometria, além de reflexo palpebral, posição do globo ocular e relaxamento muscular a cada cinco minutos.

Animais que apresentaram hipotensão transanestésica persistente, que exigisse bolus de fluidoterapia ou uso de fármacos vasoativos, foram excluídos do estudo. Variações discretas de pressão arterial eram corrigidas por meio de 
ajuste do isoflurano fornecido ou bolus intravenoso de citrato de fentanila (Fentanest®, citrato de fentanila, Cristália, Brasil) $(2 \mu \mathrm{g} / \mathrm{kg})$.

O acesso venoso foi realizado na veia safena lateral ou cefálica, antes da aplicação da medicação pré-anestésica. Para manutenção do acesso venoso, foi utilizado um dispositivo luer de acesso fechado intravenoso curto (PRN). Antes da coleta de amostras sanguíneas, era descartado, no mínimo, 0,5mL de sangue e, após a coleta, o dispositivo PRN era heparinizado com $0,5 \mathrm{~mL}$ da solução de heparina a $10 \mathrm{UI} / \mathrm{mL}$. As amostras foram armazenadas em microtubos previamente identificados e preparados com $10 \mu \mathrm{L}$ de EDTA. A fluidoterapia intravenosa teve início imediatamente antes da indução anestésica e foi interrompida ao final do procedimento cirúrgico.

Os pacientes foram submetidos a 10 coletas de sangue, cada uma de meio mililitro, em um período de 24 horas. A primeira coleta (T1) foi realizada antes da aplicação da MPA; a segunda (T2), 30 minutos após a MPA; a terceira (T3) se deu após a indução anestésica; e a quarta (T4), ao final do procedimento cirúrgico. As amostras T5, T6, T7, T8, T9, T10 foram coletadas após duas, quatro, oito, 12,18 e 24 horas do término da cirurgia, respectivamente.

Todos os animais foram submetidos à ovariossalpingo-histerectomia eletiva pela técnica das três pinças, por um único cirurgião experiente, que desconhecia o grupo a que pertenciam. O tempo cirúrgico foi padronizado em 35 a 40 minutos.

As amostras foram processadas no Laboratório de Patologia Clínica do Hospital Veterinário da Universidade Federal do Paraná - Setor Palotina, por meio de equipamento analisador hematológico automático (Mindray B62800 Vet). Após homogeneização, uma alíquota de $20 \mu 1$ foi utilizada para avaliação do hematócrito.

Os dados foram submetidos à análise de variância (ANOVA) e à comparação dois a dois pelo teste $\mathrm{t}$ entre os grupos em cada momento e à comparação pelo teste de Student NewmanKeuls (SNK) entre os tempos no mesmo grupo. Todas as comparações consideraram $\mathrm{P}<0,05$. Este estudo seguiu modelo prospectivo, randomizado e controlado.

O projeto foi certificado e obteve aprovação do Ceua local, com o protocolo de número 19/2015.

\section{RESULTADOS E DISCUSSÃO}

Todos os animais apresentaram exame físico, hemograma e exames bioquímicos préanestésicos dentro da normalidade para a espécie. Animais com sinais de anemia, leucocitose, leucopenia, trombocitopenia ou desidratação foram excluídos do estudo.

Todos os animais mantiveram valores de pressão arterial sistólica e média e frequência cardíaca dentro de parâmetros fisiológicos para a espécie e a idade durante todo o procedimento cirúrgico. Nenhum dos 12 animais apresentou hipotensão transanestésica ou bradicardia. Para todos os pacientes, o tempo cirúrgico foi de $36,67 \pm 5,16$ minutos, e o mesmo cirurgião realizou todas as cirurgias. Nenhum dos pacientes apresentou hemorragia trans ou pós-operatória.

Para G5, a média de hematócrito no momento T1 era de $48,28 \pm 7,43 \%$ e para $\mathrm{G} 10,44,12 \pm 4,75 \%$, e não foi observada diferença significativa entre os grupos (Tab. 1). Após a indução anestésica, observou-se redução significativa do hematócrito em ambos os grupos, com redução de $26 \pm 11 \%$ e $24 \pm 7 \%$ para G5 e G10, respectivamente, sem diferença numérica. Essa redução inicial do hematócrito já era esperada, pois sabe-se que fármacos vasodilatadores, como os fenotiazínicos e o propofol, podem diminuir significativamente os parâmetros hematológicos como hematócrito (Hauptman et al., 2000; Guedes et al., 2015), hemácias, hemoglobina e leucócitos (Costa et al., 2013; Picioli et al., 2013, provavelmente pelo sequestro de células em sítios não esplênicos (Costa et al., 2013). 


\section{Druziani et al.}

Tabela 1. Valores de hematócrito de cães submetidos à fluidoterapia transanestésica a 10mL/kg/h (G10) e $5 \mathrm{~mL} / \mathrm{kg} / \mathrm{h}$ (G5), avaliados por 24 horas de pós-operatório

\begin{tabular}{|c|c|c|}
\hline Tempos & G10 & G5 \\
\hline $\mathrm{T} 1$ & $44,12 \pm 4,75 \mathrm{Aab}$ & $48,28 \pm 7,43 \mathrm{Aab}$ \\
\hline $\mathrm{T} 2$ & $36,22 \pm 4,56 \mathrm{Ac}$ & $42,60 \pm 8,45 \mathrm{Aab}$ \\
\hline T3 & $33,10 \pm 3,51 \mathrm{Ac}$ & $34,92 \pm 5,98 \mathrm{Ac}$ \\
\hline $\mathrm{T} 4$ & $31,50 \pm 1,46 \mathrm{Ac}$ & $36,12 \pm 5,98 \mathrm{Ac}$ \\
\hline T5 & $30,58 \pm 5,03 \mathrm{Ac}$ & $32,97 \pm 7,76 \mathrm{Ac}$ \\
\hline T6 & $34,82 \pm 4,90 \mathrm{Ac}$ & $33,03 \pm 4,92 \mathrm{Ac}$ \\
\hline $\mathrm{T} 7$ & $34,33 \pm 3,58 \mathrm{Ac}$ & $37,02 \pm 5,94 \mathrm{Abc}$ \\
\hline $\mathrm{T} 8$ & $38,57 \pm 3,25 \mathrm{Abc}$ & $42,42 \pm 7,61 \mathrm{Abc}$ \\
\hline T9 & $38,92 \pm 6,85 \mathrm{Abc}$ & $44,58 \pm 5,90 \mathrm{Abc}$ \\
\hline $\mathrm{T} 10$ & $37,94 \pm 3,80 \mathrm{Abc}$ & $42,02 \pm 8,31 \mathrm{Abc}$ \\
\hline \multicolumn{3}{|c|}{$\begin{array}{l}\text { Tempos: (T1) imediatamente antes da pré-medicação, (T2) } 30 \text { minutos após pré-medicação, (T3) após a indução } \\
\text { anestésica, (T4) ao final do procedimento cirúrgico, (T5) após duas, (T6) quatro, (T7) oito, (T8) 12, (T9) 18, (T10) e } \\
24 \text { horas da cirurgia. Letras maiúsculas são utilizadas para mostrar possíveis diferenças na linha (entre tratamentos), e } \\
\text { letras minúsculas para mostrar possíveis diferenças significativas na coluna (entre tempos). Letras diferentes } \\
\text { representam diferença significativa. }\end{array}$} \\
\hline
\end{tabular}

Considerando que foi realizada ovariossalpingohisterectomia eletiva em pacientes hígidos, a duração da fluidoterapia transanestésica foi de curta duração. Ainda assim, observou-se redução significativa do hematócrito desde o final do procedimento cirúrgico (T4) até oito horas de pós-operatório (T7) em G10 e até quatro horas de pós-operatório (T6) em G5, evidenciando o efeito hemodiluidor mais prolongado da fluidoterapia a $10 \mathrm{~mL} / \mathrm{kg} / \mathrm{h}$. Apesar disso, não foi identificada diferença significativa entre os grupos em cada tempo. Resultado semelhante foi relatado por Gaynor et al. (1996), que compararam fluidoterapia a $0,5,10$ e $15 \mathrm{~mL} / \mathrm{kg} / \mathrm{h}$ com duração de uma hora e não observaram diferença significativa em avaliações duas horas após o procedimento cirúrgico. Greenfield et al. (1989) observaram queda de dois pontos no hematócrito ao realizarem fluidoterapia de $5 \mathrm{~mL} / \mathrm{kg} / \mathrm{h}$ por 220 minutos sem bolus prévio.

No presente estudo, foi observada redução de 12,62 pontos no hematócrito após o término da cirurgia em G10 e de 12,16 pontos em G5. É possível que a curta duração do procedimento cirúrgico e a da fluidoterapia sejam responsáveis pela pequena diferença entre os grupos, mostrando que, para curtos períodos, a velocidade de infusão da solução cristaloide não interfere significativamente no hematócrito no pós-operatório imediato.
O retorno aos valores basais de hematócrito foi determinado pela ausência de diferença significativa entre cada tempo de avaliação em comparação ao momento basal (T1). Em G10, o retorno aos valores basais foi observado 12 horas após a cirurgia (T8) em G10, quando a média do hematócrito elevou-se para 38,57 $\pm 3,25 \%$. Por outro lado, em G5 o retorno aos valores basais foi observado a partir de oito horas pósoperatório (T7), quando a média do hematócrito elevou-se para 37,02 $\pm 5,95 \%$. Apesar de Guedes et al. (2015) utilizarem fluidoterapia a $10 \mathrm{~mL} / \mathrm{kg} / \mathrm{h}$ por 120 minutos, o retorno aos valores basais de hematócrito foi observado mais precocemente quando comparado ao presente estudo, após quatro horas da cirurgia. Contudo, Guedes et al. (2015) não compararam nas mesmas condições clínicas a fluidoterapia a $5 \mathrm{~mL} / \mathrm{kg} / \mathrm{h}$, como no presente estudo, utilizando acepromazina como pré-medicação, a qual pode ter influenciado também no prolongamento da redução do hematócrito.

Fica evidente uma tendência à maior persistência da hemodiluição em G10 (Fig.1), semelhante ao relatado por Valverde et al. (2008), que evidenciaram queda de $8,6 \%$ após uma hora de fluidoterapia a $10 \mathrm{~mL} / \mathrm{kg} / \mathrm{h}$. Stamler (1989) também confirmou que $20 \mathrm{~mL} / \mathrm{kg} / \mathrm{h}$ de fluidoterapia reduzem de forma significativa até seis pontos o valor de hematócrito quando administrado por 45 minutos. 
Grupo 5

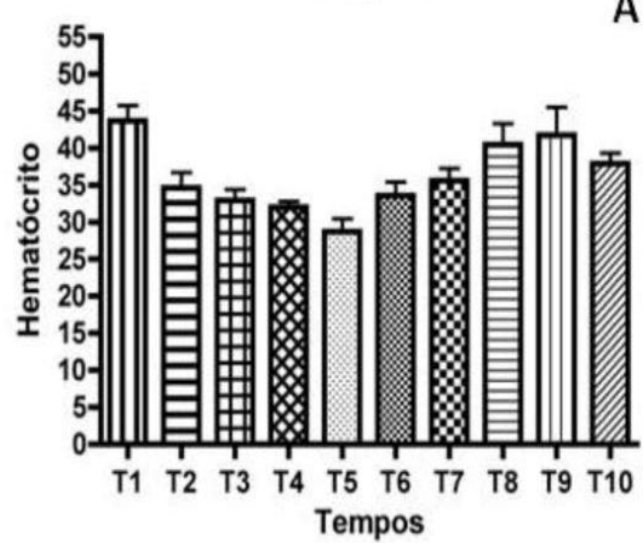

Grupo 10

B

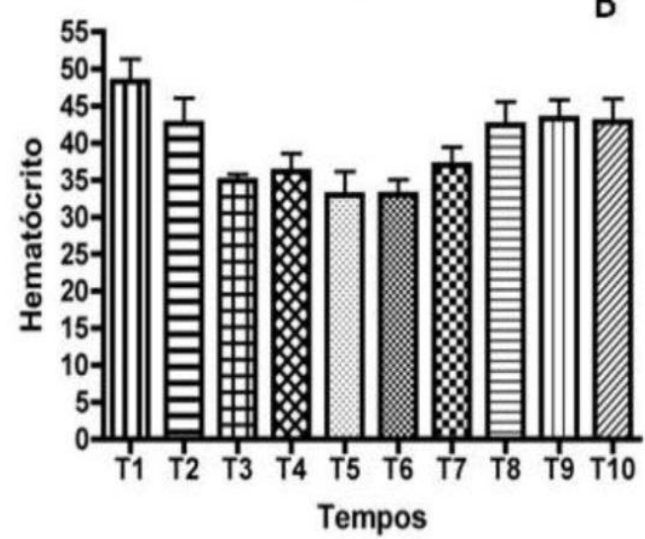

Figura 1. Valores de hematócrito de cães submetidos à fluidoterapia transanestésica. Em A, fluidoterapia a $5 \mathrm{~mL} / \mathrm{kg} / \mathrm{h}$ (G5). Em B, fluidoterapia a $10 \mathrm{~mL} / \mathrm{kg} / \mathrm{h}$ (G10). O hematócrito foi avaliado imediatamente antes da pré-medicação (T1), 30 minutos após pré-medicação (T2), após a indução anestésica (T3), ao final do procedimento cirúrgico (T4) e após duas (T5), quatro (T6), oito (T7), 12 (T8), 18 (T9) e 24 horas (T10) da cirurgia.

No presente estudo, apesar de não ter sido observada diferença entre os tratamentos, pôde-se observar redução significativa do hematócrito ao longo do tempo, com redução para valores inferiores aos de referência para a espécie (37-55\%) durante o período pósoperatório. Isso mostra que, mesmo para procedimentos de curta duração em pacientes hígidos, a fluidoterapia influencia de forma significativa no hematócrito, indicando que talvez taxas ainda menores fossem suficientes para tais procedimentos de rotina.

\section{CONCLUSÕES}

Com base nos dados encontrados no presente trabalho, pode-se concluir que não existem diferenças significativas quanto à diluição sanguínea, ao se compararem $5 \mathrm{~mL} / \mathrm{kg} / \mathrm{h}$ e $10 \mathrm{~mL} / \mathrm{kg} / \mathrm{h}$ de fluidoterapia com solução cristaloide isotônica em procedimento cirúrgico de curta duração, porém existe uma tendência à maior hemodiluição e à redução mais prolongada do hematócrito quando utilizada em $10 \mathrm{~mL} / \mathrm{kg} / \mathrm{h}$.

\section{REFERÊNCIAS}

AGUILAR-NASCIMENTO, J.E.; VALENTE, A.C.; OLIVEIRA, S.S. et al. Changes in body composition, hematologic parameters, and serum biochemistry after rapid intravenous infusion or oral intake of 2 liters of $0.9 \%$ saline solution in young healthy volunteers: randomized crossover study. World J. Surg., v.36, p.2776-2781, 2012.

CHAPPELL, D.; JACOB, M.; HOFMANNKIEFER, K. et al. A rational approach to perioperative fluid management. Anesthesiology, v.109, p.723-740, 2008.

CHOHAN, A.S.; DAVIDOW, E.D. Clinical pharmacology and administration on fluid, eletrolyte and blood component solutions. In: GRIMM, A.K.; LAMONT, L.A.; TRANQUILLI, W.J. et al. Veterinary anesthesia and analgesia. 5.ed. St.Louis: Willey-Blackwell, 2015. p.386-417.

COSTA, P.F.; NUNES, N.; BELMONTE, E.A. et al. Hematologic changes in propofolanesthetized dogs with or without tramadol administration. Arq. Bras. Med. Vet. Zootec., v.65, p.1306-1312, 2013. 
DAVIS, H.; JENSEN, T.; JOHNSON, A. et al. AAHA/AAFP fluid therapy guideline for dogs and cats. J. Am. Anim. Hosp. Assoc., v.49, p.149$159,2013$.

FUNK, W.; BALDINGER, V. Microcirculatory perfusion during volume therapy: a comparative study using crystalloid or colloid in awake animals. Anesthesiology, v.82, p.975-982, 1995.

GAYNOR, J.S.; WERTZ, E.M.; KESEL, L.M. et al. Effect of intravenous administration of fluids on packed cell volume, blood pressure, and total protein and blood glucose concentrations in healthy halothane-anesthetized dogs. J. Am. Vet. Med.Assoc., v.208, p.2013-2015, 1996.

GREENFIELD, R.H.; BESSEN, H.A.; HENNEMAN, P.L. Effect of crystalloid infusion on hematocrit and intravascular volume in healthy, nonbleeding subjects. Ann. Emerg. Med., v.18, p.51-55, 1989.

GUEDES, R.L.; CASTRO, V.S.P.; PALMA, E.H. et al. Influence of Ringer's lactated solution in continuous infusion and general anesthesia on hematocrit in dogs. Semin. Ciênc. Agrár., v.36, p.2679-2684, 2015.

HALL， L.W.; CLARK, K.W.; TRIM， C.M. Anaesthesia of the dog. In: Hall LW, Clarke KW, Trim CM, editor. Veterinary anaesthesia. 10 ed. Londres: Saunders, 2001. p.385-439.
HAUPTMAN, J.G.; RICHTER, M.A.; WOOD, S.L.; NACHREINER, R.F. Effects of anesthesia, surgery, and intravenous administration of fluids on plasma antidiuretic hormone concentrations in healthy dogs. Am. J. Vet. Res., v.61, p.12731276, 2000.

MATHEWS K.A. Monitoring fluid therapy and complications of fluid therapy. In: DI BARTOLA, S.P. Fluid, electrolyte, and acidbase disorders in small animal practice. 3.ed. St. Louis: Saunders, 2006. p.377-391.

PICIOLI, A.; MARTINI, M.V.; MINERVINO, A.H.H. et al. E. O uso da acepromazina, dexmedetomidina e xilazina na sedação em cães: alterações hematológicas e bioquímicas. Rev. Bras. Cienc. Vet., v.20, p.13-19, 2013.

STAMLER,K.D. Effect of crystalloid infusion on hematocrit in nonbleeding patients, with applications to clinical traumatology. Ann. Emerg. Med., v.18, p.747-749, 1989,.

VALVERDE, A.; HATCHER, E.; STAMPFLI, H.R. Effects of fluid therapy on total protein and its influence on calculated unmeasured anions in the anesthetized dog. J. Vet. Emerg. Crit. Care, v.18, p.480-487, 2008. 